\title{
Ultrasound assisted low temperature drying of food materials
}

\author{
Sabarez, H.T. ${ }^{\text {a*}}$; Keuhbauch, S. ${ }^{\text {b; Knoerzer, K. }}{ }^{\text {a }}$ \\ ${ }^{\text {a }}$ CSIRO Agriculture \& Food, Werribee, Victoria, Australia \\ ${ }^{\mathrm{b}}$ Karlsruhe Institute of Technology (KIT), Kaiserstrabe 12, 76131 Karlsruhe, Germany \\ *E-mail of the corresponding author: henry.sabarez@csiro.au
}

\begin{abstract}
An ultrasonic design based on the indirect transmission of ultrasonic energy from the ultrasound emitter through to the material to be dried was investigated to assist in low temperature drying of food materials. The application of the improved design tested in this work was found to enhance the low temperature drying by shortening the overall drying time of up to $45 \%$ (i.e., lower energy consumption and may enable better retention of product quality). This offers a promising approach towards a better applicability of ultrasound in industrial operation, since no direct contact between the sample and the ultrasonic emitter is needed.
\end{abstract}

Keywords: ultrasound; drying; low temperature; drying intensification. 


\section{Introduction}

Many drying techniques evolved due to the need to produce high quality products that are highly heat-sensitive. Such drying systems include the utilization of low temperatures, but often require a very long drying time, highly energy consuming and detrimental to the product quality. These limitations can be overcome by the combined application of ultrasound, which has been demonstrated in previous studies to intensify the convective drying processes. ${ }^{[1,2]}$ However, the development of ultrasound assisted drying technology at an industrial scale has progressed at a slow pace due to the difficulties in achieving an efficient transmission of ultrasonic energy from the plate transducers to the product while ensuring easy adaptability to conventional drying processes.

The ultrasonic systems reported in the literature for food drying application may be grouped into two main types. The first type is made up of ultrasonic devices that are directly coupled to the food material during drying. The direct contact system can promote an accelerated drying process because this system permits good transfer of ultrasonic energy from the vibrating element to the food material. Nevertheless, the main drawback of this technique may be its difficulty to adapt to traditional airflow drying processes and in operation other than a batch-wise process.

Another type was developed based on the application of airborne ultrasound. The airborne ultrasonic system works without direct contact between the vibrating element and the food material, which seems to offer much better adaptability to conventional air drying processes. However, the main difficulties in this system arise from the inefficient generation of ultrasonic energy in air and the transfer of such ultrasonic energy from air into the product due to the acoustic impedance mismatch and the energy absorption by the air at ultrasonic frequencies. ${ }^{[3]}$

The aim of this work was to develop and test an improved design concept for an effective application of ultrasound at high frequencies that would allow easy adaption in continuous processing while facilitating efficient ultrasonic energy transmission to intensify low temperature drying of food materials.

\section{Materials and Methods}

Fresh apples (cv. Granny Smith) obtained from a local market (Werribee, Victoria, Australia) were used as the model test material and were stored at $4^{\circ} \mathrm{C}$ until further processing. The sample preparation has been described in detail by Sabarez et al. ${ }^{[2]}$ The fresh apple samples were found to have an average initial moisture content of $86.0 \%$ (wet basis). 
Drying experiments were carried out in a computerised ultrasound-assisted convective drying setup as described elsewhere ${ }^{[2,4]}$, which had been modified to retrofit the improved ultrasound transmission system (Fig. 1). The ultrasonic transmission system consisted of a transmission platform, cooling unit (Model BL-130, Thermoline, Australia) to control the temperature of the transmission platform, and a plate transducer using Blackstone-Ney Ultrasonic Generator (Blackstone-Ney Ultrasonics Inc., Jamestown, New York, USA).

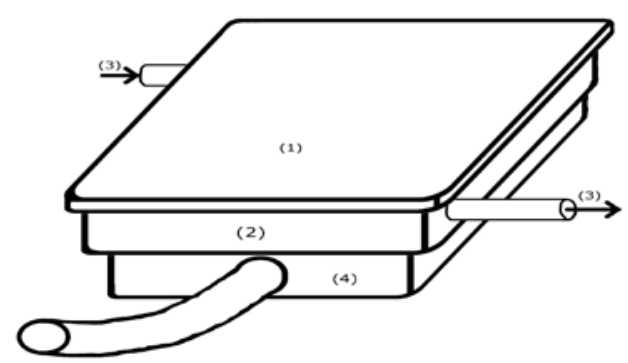

Fig. 1. Ultrasound transmission setup (1=transmission plate; $2=$ frame for containing the cooling/heating liquid; 3=cooling/heating liquid; 4=transducer).

The advantage of this experimental drying system is that it consists of a number of additional sensors (i.e., thermocouples, infrared noncontact temperature sensors, air velocity sensors, humidity sensors, etc.) that are interfaced to a computer-based data acquisition and control system for continuous online monitoring and recording of the various processing conditions (i.e., drying time, material temperatures, air temperature, air relative humidity, and air velocity).

Two sets of drying experiments were carried out without ultrasound and with ultrasound at a frequency of $40 \mathrm{kHz}$. In these experiments, the apple ring samples were directly placed on the transmission platform. Four annular apple rings prepared as outlined above were used for each drying experiment. All drying experiments were replicated at least twice. The details of the drying procedure can be found elsewhere. ${ }^{[2,4]}$

\section{Results and Discussion}

Figure 2 shows a typical example of the drying kinetics during the convective air drying of $5 \mathrm{~mm}$ apple samples (about $100 \mathrm{~g}$ ) at $40^{\circ} \mathrm{C}$ temperature (T) without and with ultrasound at $40 \mathrm{kHz}$ frequency with power level of $466 \mathrm{~W}$. These experiments were conducted with drying air velocity (V) and relative humidity (RH) maintained at about $1.2 \mathrm{~m} / \mathrm{s}$ and 25\%, respectively. 
It is clear from Figure 2 that the application of ultrasound in combination with convective air drying significantly reduced the overall drying time. To achieve the target moisture content of $25 \%$ in wet basis (i.e, typical moisture content of ready-to-eat fruit snacks), analysis of the drying curves revealed that it took about 8.0 hours to dry the apple samples without ultrasound and just 4.4 hours with ultrasound using the improved ultrasonic transmission system. The results indicate a significant reduction in drying time of about $45 \%$ with the simultaneous application of ultrasound on the convective drying of apple slices (corresponds to a 32\% reduction of energy consumption).

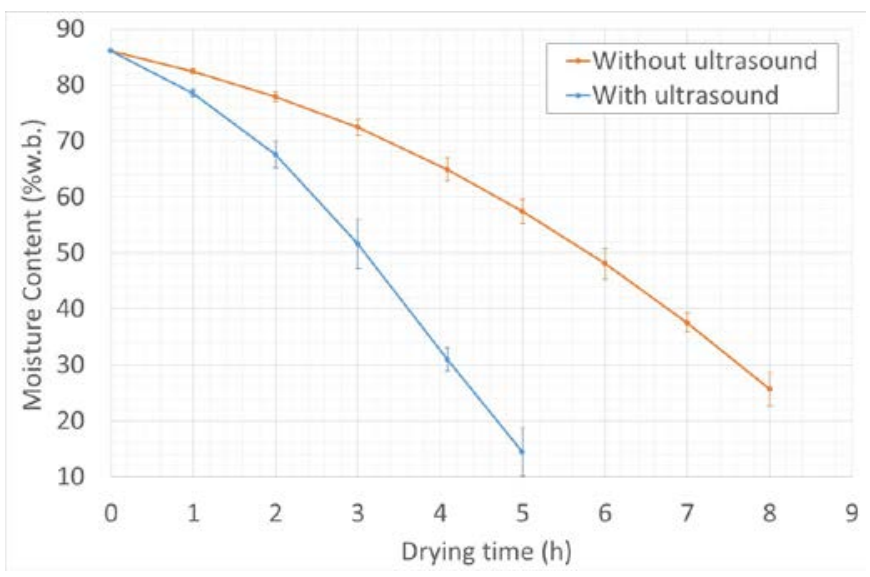

Fig. 2. Effect of ultrasound (40 $\mathrm{kHz}$ frequency; $466 \mathrm{~W}$ power) on the drying kinetics of apple slices ( $T=40^{\circ} \mathrm{C} ; \mathrm{RH}=25 \% ; \mathrm{V}=1.2 \mathrm{~m} / \mathrm{s} ; 5 \mathrm{~mm}$ thickness).

The plot clearly shows that the ultrasonic transmission system developed and tested in this work was highly effective in intensifying the convective drying process. It also demonstrates that high frequency ultrasound can assist in enhancing the convective drying process. Although most of the physical mechanisms by which low frequency ultrasound enhanced the drying process are highly suppressed at high frequency, the observed enhancement in drying with high frequency ultrasound may be explained by Stokes' law of attenuation. Higher frequency ultrasound undergoes more attenuation, inducing more microstreaming (a non-thermal effect that occurs due to a pressure gradient generated by aborption of ultrasound during propagation) thereby increasing the diffusivity of water. So far, no studies have been reported on the application of ultrasound at higher frequencies in assisting food drying processes. Most of the studies were carried out at low frequencies (20$26 \mathrm{kHz}$ ) with drawbacks of low energy efficiency and high noise levels, with the ultrasonic 
energy transmitted either in direct contact between the vibrating plate and the product ${ }^{[5,6]}$ or airborne to the surface of the product. ${ }^{[2,3,4,7,8]}$ These studies have reported significant reductions in drying time with the application of ultrasound, depending on ultrasonic parameters, drying conditions and properties of the materials to be dried.

Sabarez et al. ${ }^{[2]}$ found a significant reduction in drying of up to $57 \%$ with the application of airborne ultrasound during drying of $5 \mathrm{~mm}$ apple samples. Under these drying conditions, an approximately up to 54\% reduction in energy consumption during drying could be achieved with the application of airbone ultrasonic energy. Similarly, Garcia-Perez et al. ${ }^{[7]}$ found a significant reduction in drying time (up to $70 \%$ at acoustic power of $90 \mathrm{~W}$ ) with the application of power ultrasound for convective drying of eggplant cylinders. Ortuno et al. ${ }^{[9]}$ observed that the application of ultrasound (power level of $90 \mathrm{~W}$ ) provided an average reduction in drying time of over $45 \%$ for drying of orange peels. They found that the energy saving was close to $30 \%$ with ultrasonic application.

The differences in drying time reduction and energy saving between these studies and the present work could be due to the differences in the raw material properties, drying conditions and ultrasonic parameters (i.e., particularly ultrasonic frequency, power and mode of ultrasound transfer). In the present study, high frequency ultrasound is transmitted indirectly through to the transmission platform (liquid and steel) and into the material to be dried. This allows for an efficient transmission of ultrasound to the material to be dried as the mismatch of acoustic impedances of these materials (steel, liquid and food) is minimized, and would enable for operation in conjuction with traditional air drying, along with easy integration in continuous processing as there is no direct contact between the ultrasonic emitter and the food samples to be dried.

\section{Conclusions}

The application of an improved ultrasound transmission system was found to significantly intensify the low temperature air drying of apple samples by reducing the overall drying time (i.e., lower energy consumption and may enable better retention of product quality). This offers a promising non-thermal means for gentle (i.e., low temperature) drying of food materials to produce premium quality food products, with better applicability at industrial scale as no direct contact between the food material and ultrasonic transducer is needed. In addition, the present study demonstrates that high frequency ultrasound can assist in enhancing convective drying with much reduced noise levels. The results from this work will provide the basis to build upon the development of this technology and justifies further investigations for implementation in industrial drying operations. 


\section{References}

[1] Sabarez, H.T. Airborne ultrasound for convective drying intensification. In Innovative Food Processing Technologies - Extraction, Separation, Component Modification and Process Intensification; Knoerzer, K., Juliano, P., Smithers, G., Eds.; Woodhead Publishing, An Imprint of Elsevier, Elsevier B.V., 2016; 361-386.

[2] Sabarez, H.T.; Gallego-Juarez, J.A.; Riera, E. Ultrasonic-assisted convective drying of apple slices. Drying Technology 2012, 30 (9), 989-997.

[3] Gallego-Juarez, J.A.; Rodriguez-Corral, G.; Galvez-Moraleda, J.C.; Yang, T.S. A new high intensity ultrasonic technology for food dehydration. Drying Technology 1999, 17 (3), 597-608.

[4] Beck, S.M.; Sabarez, H.T.; Gaukel, V.; Knoerzer, K. Enhancement of convective drying by application of airborne ultrasound: a response surface approach. Ultrasonics Sonochemistry 2014, 21, 2144-2150.

[5] Gallego-Juarez, J.A.; Riera, E.; de la Fuente Balanco, S.; Rodriguez-Corral, G.; Acosta-Aparicio, V.M.; Blanco, A. Application of high-power ultrasound for dehydration of vegetables: Processes and Devices. Drying Technology 2007, 25(11), 1893-1901.

[6] Schossler, K.; Jager, H.; Knorr, D. Effect of continuous and intermittent ultrasound on drying time and effective diffusivity during convective drying of apple and red bell pepper. Journal of Food Engineering 2012, 108 (2012), 103-110.

[7] Garcia-Perez, J.V.; Carcel, J.A.; Riera, E.; Mulet, A. Influence of the applied acoustic energy on the drying of carrots and lemon. Drying Technology 2009, 27(2), 281-287.

[8] Kowalski, S.J.; Pawlowski, A. Intensification of apple drying due to ultrasound enhancement. Journal Food Engineering 2015, 156, 1-9.

[9] Ortuno, C.; Perez-Munuera, I.; Puig, A.; Riera, E.; Garcia-Perez, J.V. Influence of power ultrasound application on mass transport and microstructure of orange peel during hot air drying. Physics Procedia 2010, 3, 153-159. 